\title{
Substance Use Among Older Adults with Bipolar Disorder Varies According to Age at First Treatment Contact
}

Osvaldo P. Almeida ${ }^{1,2,3}$

Graeme J. Hankey ${ }^{1,4}$

Bu B. Yeap ${ }^{1,5}$

Jonathan Golledge $e^{7,8}$

Leon Flicker ${ }^{1,2,9}$

${ }^{1}$ Medical School, University of Western Australia, Perth, Australia

${ }^{2}$ WA Centre for Health \& Ageing of Centre for Medical Research, Harry Perkins Institute of Medical Research, Perth, Australia

${ }^{3}$ Department of Psychiatry, Royal Perth Hospital, Perth, Australia

${ }^{4}$ Department of Neurology, Sir Charles Gairdner Hospital, Perth, Australia

5 Department of Endocrinology and Diabetes, Fiona Stanley Hospital, Perth, Western Australia

7 Queensland Research Centre for Peripheral Vascular Disease, College of Medicine and Dentistry, James Cook University, Townsville, Australia

${ }^{8}$ Department of Vascular and Endovascular Surgery, The Townsville Hospital, Townsville, Australia

${ }^{9}$ Department of Geriatric Medicine, Royal Perth Hospital, Perth, Australia

Running title: Older Bipolar and Substance Use

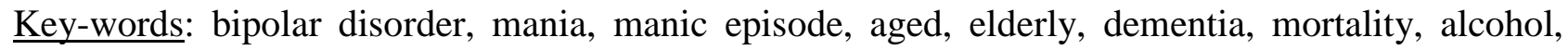
substance use disorder, epidemiology.

Word count: 3985

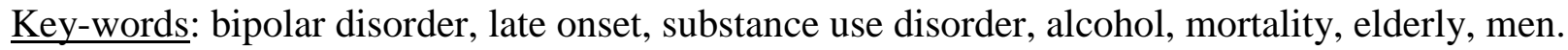

\section{Correspondence}

Prof. Osvaldo P. Almeida, Medical School (M573), University of Western Australia, 35 Stirling Highway, Crawley, Perth, WA 6009, Australia. Email: osvaldo.almeida@uwa.edu.au. 


\section{INTRODUCTION}

The use of substances, such as alcohol, is common among people living with bipolar disorder (BD). Data from the Epidemiologic Catchment Area (ECA) study showed that $43.6 \%$ and $33.6 \%$ of adults with BD had experience a comorbid alcohol or drug use disorder respectively during their lifetime, a prevalence estimate that is 5 to 8 times greater than for people without a mental disorder (Regier et al., 1990). Subsequent studies have confirmed the association between the use of substances and BD,(Hunt et al., 2016) which is concerning because this association increases health morbidity and mortality (Whiteford et al., 2013), including death by suicide (Cardoso et al., 2008; Dalton et al., 2003).

The relationship between $\mathrm{BD}$ and the use of substances is complex and not entirely clear. It is possible that the psychological and behavioural features that characterise BD lead to an increase in the use of substances (Swendsen et al., 2010), or that people with BD use substances as an attempt to manage affective symptoms (Meyer et al., 2012). If that is the case, it is possible that older people with BD may present a different pattern of use of substances, as coping and defence mechanisms change with age. Existing evidence suggests that younger adults are more prone to using outward and undifferentiated strategies, while older adults show greater impulse control and a tendency to positively appraise situations associated with conflict (Diehl et al., 1996). Preliminary cross-sectional data from the National Epidemiologic Survey on Alcohol and Related Conditions (NESARC) showed that the odds of alcohol use disorders during the preceding 12 months were nearly twice as low among BD participants older than, compared with younger than, 65 years of age, although a cohort effect on the results could not be discarded (Goldstein et al., 2006). This example of the apparent contrast between the clinical presentation of young and older adults with $\mathrm{BD}$ has led to the suggestion that there may be merit in grouping people with $\mathrm{BD}$ according to the age at first treatment contact of symptoms (Almeida et al., 2016; Sajatovic et al., 2015). The OlderAge Task Force of the International Society for Bipolar Disorders reported that there was consensus 
that 60 years is an appropriate age cut-point to define late onset BD (Sajatovic et al., 2015), which may be relevant when considering the use of alcohol and other substances.

In order to minimise the potential cohort effect associated with the comparison of different age groups at one point in time, it is important to investigate the association between the use of alcohol and other substances and BD in a community-representative sample of older adults that includes people with early and late onset of illness. In this instance, we would anticipate that lifetime substance use disorders would be more prevalent among older adults whose BD started early rather than later in life. In addition, if we consider that the use of substances is primarily driven by the age of onset rather than BD itself, then the incidence of substance use disorders in a sample of older people should be independent of the age of onset of symptoms (i.e., older adults with BD would be expected to use similar adaptive strategies to cope with symptoms). Clarifying these associations is more than an academic exercise: substance use disorders complicate the course of BD (Hjorthoj et al., 2015) and, given the rapid ageing of the population, it is important to clarify if the increasing number of older adults with BD require an approach to treatment that addresses both conditions.

This study aimed to: (1) examine the cross-sectional association between the prevalence of disorders associated with the use of alcohol or other substances with the age at first treatment contact of $\mathrm{BD}$; (2) determine the longitudinal association between age at first treatment contact of BD and incident disorders associated with the use of alcohol or other substances; (3) investigate the mortality hazard associated with age at first treatment contact of $\mathrm{BD}$ and how this might be modified by the presence of alcohol or other substance use disorder.

\section{METHODS}

Study design and setting 
This report consists of a cross-sectional and a cohort study of community-dwelling older men living in the Perth metropolitan region of Western Australia.

\section{$\underline{\text { Participants }}$}

We used the Australian Electoral Roll (voting is compulsory for all Australian citizens aged 18 years or over) to identify all Australian men aged 65-85 living in the Perth metropolitan region during 1996-1998. We identified 49801 potentially men. Of these, 1839 had died by the time the study started, 9482 were living outside the study region and 307 were younger than 65 years (these men were invited in error). Hence, the study sample consisted of 38173 older men. This cohort was recruited to investigate the effect of screening for aortic aneurysms on mortality. Only men were recruited because aortic aneurysm is much more frequent in men than women. These participants were then de-identified before the retrieval of clinical information (please see details below). The Ethics Committees of the University of Western Australia and of the Department of Health of Western Australia approved the study protocol and procedures.

\section{$\underline{\text { Study measures }}$}

We used the Western Australian Data Linkage System (WADLS) to obtain all available clinical information about participants. WADLS links health service data (including dates of contact) from inpatient and outpatient mental health services, hospital morbidity data, community aged care services, as well as cancer and death registries (Holman et al., 2008). The system uses the International Classification of Diseases (ICD) system for the coding of clinical diagnoses and procedures: ICD-8 from $1^{\text {st }}$ January 1966 to $31^{\text {st }}$ December 1969 , ICD-9 from $1^{\text {st }}$ January 1970 to $30^{\text {th }}$ June 1999 , and ICD-10 from the $1^{\text {st }}$ July 1999. WADLS allows for the logging of multiple diagnoses at each contact. 
We used the following ICD codes to establish the presence of BD: 296.1 and 296.3 (ICD-8), 296.0, 296.1, 296.4, 296.5, 296.6, 296.7, 296.80 and 296.81 (ICD-9), and F30 or F31 (ICD-10). We considered that the date of the first ever episode of bipolar disorder was the same as the date of the first ever contact with the health services with one of these diagnoses (outpatient or inpatient). If a depressive episode had been recorded before an episode of mania or mixed state, then the date of the depressive episode was considered the date of onset of BD. The ICD codes that identified depressive episodes were: 296.0 and 300.4 (ICD-8), 296.2, 296.3, 311 and 300.4 (ICD-9), and F32, F33, F34.1 and F38.10 (ICD-10). The earliest mental health morbidity records available were dated $1^{\text {st }}$ January 1966, so that the minimum age of BD onset recordable would have been 35 years (i.e., 30 years before the start of the study). We used a hierarchical approach to diagnosis, so that participants who received a diagnosis of schizophrenia or delusional disorder at any point in time (ICD codes 295, 297, F20, F22, F23, F25, F28 and F29) were considered not to have BD. We defined late onset BD as a disorder with onset at or after the age of 60 years (Sajatovic et al., 2015).

Alcohol and other substance use disorders represented the main outcomes of interest of the study. The ICD codes that identified alcohol use disorders were: 291 and 303 (ICD-8), 291, 303, 305.0 (ICD-9) and F10 (ICD-10). The codes for other substance use disorders were 304 (ICD-8), 292, 304, 305 (excluding 305.0) (ICD-9), and F11 to F19 (ICD-10). These codes include disorders (dependence, withdrawal or intoxication) due to the use of opioids, cannabinoids, sedatives or hypnotics, cocaine, stimulants (including caffeine), hallucinogen, tobacco, volatile solvents and other psychoactive substances.

Other study measures included diabetes (ICD-8 and 9 codes 249 and 250, and ICD-10 codes E08 to E13), hypertension (ICD-8 and 9 codes 410 to 413 and ICD-10 codes I10 and I12), ischaemic heart disease (ICD-8 and 9 codes 410 to 414, and ICD-10 codes I20 to I25) and stroke (ICD-8 and 9 codes 430 to 434, 436 to 438, and ICD-10 codes I60 to I69). We calculated the age of participants 
in years by subtracting the date of contact from the date of their birth and then dividing the result by 365.25. We also used WADLS to ascertain the date of death of participants.

\section{$\underline{\text { Statistical analyses }}$}

We used the statistical software Stata 15.1 to manage and analyse the data (StataCorp LLC, Revision 11 January 2018). Descriptive statistics summarised categorical variables as count and proportions (\%), and continuous variables as mean, range, and standard deviation of the mean (SD). We used oneway analysis of variance to compare the ages of participants according to the age at first treatment contact of bipolar disorder. We then used multinomial logistic regression to investigate the association between BD and concurrent morbidities and adjusted all analyses for the effect of age (in years) - risk estimates were expressed as odds ratio (OR). Finally, we used Cox regression (Breslow method) to investigate the hazard ratio (HR) of incident disorders associated with the use of alcohol or other substance use and death. In these models, we split and joined timespan sets according to the diagnosis of BD (including the age at first treatment contact), so that men without BD contributed data as controls until the time of diagnosis and as cases thereafter. We used age as the time scale in the Cox regression models in order to control as accurately as possible the effect of age on the use of substances and mortality.(Breslow et al., 1983) Alpha was set at 5\% and all risk estimates were reported alongside their respective $95 \%$ confidence interval $(95 \% \mathrm{CI})$.

\section{RESULTS}

The age of the 38173 participants at study entry ranged from 65 to 85 years (mean \pm SD $=72.5 \pm$ 4.6). Of these, $250(0.6 \%)$ had a prevalent diagnosis of BD, $1541(4.0 \%)$ of alcohol use disorder and $6070(15.9 \%)$ of another substance use disorder. 707 (1.9\%) men had a recorded diagnosis of both alcohol and another substance use disorder. There was an excess of cases of alcohol and other substance use disorders among older men with $\mathrm{BD}(\mathrm{n}=33 / 250, \mathrm{OR}=3.67,95 \% \mathrm{CI}=2.54,5.32$ and $\mathrm{n}=63 / 250, \mathrm{OR}=1.79,95 \% \mathrm{CI}=1.34,2.38$, respectively). The respective prevalence of any substance 
use disorder was $32.4 \%$ and $18.0 \%$ for men with and without BD (OR of substance use disorder for men with relative to men without $\mathrm{BD}=2.18,95 \% \mathrm{CI}=1.64,2.96)$.

Table 1 shows the age distribution and that of disorders associated with the use of alcohol and of other substances according to the age at the time of first diagnosis of $\mathrm{BD}$ (age at onset). The groups differed in age at study entry $(\mathrm{F}=13.66, \mathrm{df}=2, \mathrm{p}<0.001)$. Men with $\mathrm{BD}$ onset $<60$ years were younger than all other participant groups $(\mathrm{p}<0.01)$, whereas those with BD onset $>60$ years were older than all others $(\mathrm{p}<0.01)$. The odds of alcohol use disorder were increased for men with BD regardless the age at first treatment contact, although the risk estimate declined with increasing age at first treatment contact. The odds of other substance use disorder were only significantly increased for men with $\mathrm{BD}$ onset $<60$ years.

\section{TABLE 1}

Compared with men without BD, men with $\mathrm{BD}$ (both with onset before and after age 60 years, $\mathrm{n}=250)$ had higher prevalence of diabetes $(7.7 \%$ vs $13.2 \%)$ and past strokes (6.9\% vs $11.2 \%)$, and a non-significant excess of hypertension $(26.6 \%$ vs $29.2 \%)$ and ischaemic heart disease $(23.5 \%$ vs 27.6\%), respectively. Diabetes, hypertension, ischaemic heart disease and strokes were all more frequent among men with than without an alcohol or other substance use disorder $(\mathrm{p}<0.001$ for all comparisons). For this reason, these variables, alongside with age, were included in all models investigating the prospective association between BD and substance use disorders.

During the follow up period of up to 17.7 years, 24378 (63.9\%) men died, of whom 206 had BD. The mortality hazard was higher among participants with than without $\mathrm{BD}(\mathrm{HR}=1.38,95 \% \mathrm{CI}=1.18$, 1.56). During this time, 1099 new cases of alcohol use disorder and 1512 of other substance use disorder were recorded. Figure 1 shows the cumulative hazard of incident alcohol use disorder 
associated with the diagnosis of BD according to age at onset. The HR of alcohol use disorder was $3.23(95 \% \mathrm{CI}=1.87,5.58)$ and $1.81(95 \% \mathrm{CI}=0.75,4.35)$ for participants with bipolar disorder with onset before or at/after 60 years, respectively. The risk estimates were adjusted for age and prevalent diabetes, hypertension, ischaemic heart disease and stroke. Similarly, the adjusted HRs of incident disorders associated with the use of substances other than alcohol were 2.38 (95\% $\mathrm{CI}=1.38$, 4.11) and $0.74(95 \% \mathrm{CI}=0.24,2.31)$ for men with $\mathrm{BD}$ onset $<60$ years and $\geq 60$ years, respectively.

\section{FIGURE 1}

Finally, we calculated the mortality hazard associated with age at onset of BD and the disorders associated with the use of alcohol and other substances. Compared with men without BD, the adjusted mortality hazard of participants with BD was higher, independent of their age at onset of symptoms $(\mathrm{HR}=1.33,95 \% \mathrm{CI}=1.10,1.61$ and $\mathrm{HR}=1.42,95 \% \mathrm{CI}=1.09,1.85$ for men with $\mathrm{BD}$ onset $<$ 60 years and $\geq 60$ years, respectively). Similarly, men with a recorded diagnosis of alcohol use disorder had higher adjusted mortality hazard than those without $(\mathrm{HR}=2.01,95 \% \mathrm{CI}=1.90,2.13)$, but the interaction between alcohol and age at onset of BD did not significantly change mortality over time ( $>0.05)$. Likewise, the diagnosis of a disorder associated with the use of substances other than alcohol increased the adjusted mortality hazard $(\mathrm{HR}=1.49,95 \% \mathrm{CI}=1.44,1.54)$, but did not interact with age at onset of bipolar disorder to modulate the risk of death over time ( $>>0.05)$.

\section{DISCUSSION}

The results of this study showed that the prevalence of alcohol use disorder among older men with BD was $13.2 \%$, nearly 4 times as high as in men without $\mathrm{BD}$, while the prevalence of a disorder associated with the use of substances other than alcohol was $25.2 \%$ among participants with BD, nearly twice as high as the remainder of the population. One in every 3 older men with BD had received a diagnosis of a substance use disorder by the time of recruitment into the study, although 
the prevalence of disorders associated with the use of alcohol and of other substances declined with increasing age at first treatment contact of $\mathrm{BD}$ and became non-significant for substances other than alcohol. Compared to men without BD, the incidence of disorders associated with the use of alcohol and other substances was increased for older men with onset of BD before age 60 years, but not after. We also found that both BD and disorders associated with the use of substances (alcohol or other) increased the risk of death among participants, but their effects were independent from one another.

Before discussing the implications of these results, we will consider the characteristics of the study design and how these might have affected the validity and generalisability of our findings. We recruited a large community-representative sample of older men in the metropolitan region of Perth, Australia. The sample of nearly 40000 people was sufficiently large to investigate associations with low-prevalence conditions, such as BD. This also allowed us to investigate the impact of age at first treatment contact of BD on the prevalence and incidence of substance use disorders. In addition, WADLS enabled us to track the health movements of participants before and after the start of the study, which in turn ensured that loss to follow up would be kept to a minimum as the migratory movement of older Western Australians is negligible (ABS, 2015).

The diagnoses of BD and of alcohol and other substance use disorders were retrieved from WADLS, which uses administrative clinical data rather than a structured clinical assessment. We attempted to minimise misclassification by adopting a hierarchical approach to diagnosis, and this seems to have produced prevalence estimates that are consistent with those produced by surveys using a different methodology (Depp and Jeste, 2004; Merikangas et al., 2011; Sajatovic et al., 2006; Schouws et al., 2009). In addition, others have shown that WADLS yields valid diagnoses for affective disorders and schizophrenia (Jablensky et al., 2005), which offers some confidence that our approach to the assessment of the exposure was valid. We acknowledge, however, that the 
establishment of the onset of BD was based on the age of first contact with the health services rather than the age when symptoms first appeared. While nearly all people who experience manic symptoms come in contact with the health services in Western Australia, it is possible that mild episodes of depression occurring prior to the onset of mania or hypomania would not have been recorded. Hence, some misclassification of 'late onset' cases may have occurred. Such a bias would have resulted in dilution of the effects associated with age at first treatment contact and type II error. We also concede that WADLS only started tracking records after 1966, so that early health contacts would have not been recorded. Consequently, none of our men could have been ascribed an age at first treatment contact earlier than 35 years. Nonetheless, it seems improbable that many of our participants with onset of BD in their late teens or 20 s would have reached the age of 50 years without having experienced an additional affective episode, so that the misclassification of 'early onset' cases as 'late onset' would seem improbable (Coryell et al., 2013).

The validity of the diagnoses of alcohol and other substance use disorders obtained from WADLS are less clear than that of $\mathrm{BD}$ and, conceivably, could be biased towards the more severe end of the spectrum. This issue is compounded by the lack of information about the severity of the use of alcohol or other substances. We were also unable to disentangle cases of intoxication from abuse and dependence, as available WADLS coding often failed to provide additional specifiers. This lack of specificity regarding the diagnosis of substance use disorders may have been particularly pronounced in the case of substances other than alcohol, which could explain their high prevalence in our sample. We accept that the category 'other substance use disorder' may have been overinclusive (e.g., caffeine, sedatives), but we concluded that we should maintain this group unchanged because we wished to explore the pattern of substance use among older men with BD rather than the use of a particular substance itself. Moreover, the relatively modest number of people with BD in the sample would have limited our ability to complete multiple comparisons of all possible associations. Notwithstanding these concerns, the prevalence of alcohol use disorders in our study 
was consistent with that of other reports (O'Connell et al., 2003), which suggests that, at least in the case of alcohol, WADLS may have been effective at identifying cases. We are also mindful of the fact that men with BD may be in contact with health services more frequently than men without $\mathrm{BD}$, and this could increase the opportunity for the diagnosis of a substance use disorder to be established. This type of bias could potentially inflate the association between BD and the use of substances (a form of Berkson's bias). While we cannot dismiss such a possibility entirely, the strength of the associations observed suggest that bias alone is an unlikely explanation for the results. In addition, health contacts increase with increasing age (because of comorbidities and frailty), so that age becomes the driving force behind opportunistic case finding in later life. In our cohort, older late onset BD participants did not show an increased risk of incident disorders associated with the use of substances, suggesting that the general findings of the study are most likely valid. Finally, we are mindful of the fact that our results describe clinical associations among older men, but not women, although others have reported similar prevalence of alcohol use disorders among older Australian women (Clemens et al., 2007).

As expected, we found that disorders associated with the use of alcohol and other substances were more prevalent among older adults with than without $\mathrm{BD}$, and were more frequent among men with onset of BD before age 60 years. This finding could be interpreted as indicating that BD interacts with age to modulate the risk of use of substances, with this risk decreasing as the age at first treatment contact of symptoms increases. However, our cross-sectional data could not offer evidence for causality or even for the direction of the association between BD and substance use disorders. Our prospective data contribute to address this issue: we found that BD increases the risk of incident substance use disorders in older men (both alcohol and other substances). This increase in risk was limited to people who had experienced the onset of affective symptoms before age 60 years. Both the cross-sectional and longitudinal data arising from this study lend support to the 
suggestion that grouping BD according to age at first treatment contact (early vs late) is clinically meaningful (Almeida et al., 2016; Sajatovic et al., 2015).

But why would the association between BD and substance use disorders affect preferentially older adults with BD onset $<60$ years? One possibility is that the pathophysiological mechanisms associated with early and late onset BD are not exactly the same. Preliminary evidence suggests that manic and hypomanic symptoms may arise in association with certain neurodegenerative diseases (Cerami et al., 2011; Floris et al., 2013), and this could lead to a different course of the clinical presentation over time. However, other studies have shown that the course of BD and the associated causes of death are not affected by the age at first treatment contact of symptoms (Almeida et al. under review), which suggests that there may be more similarities than differences between early and late onset cases in this regard. Alternatively, the association between BD and substance use could be driven by the duration of the disorder, so that older people with early onset of symptoms would have more prolonged exposure to BD than those with late onset. We are not aware of any compelling evidence that the duration of BD modulates the risk of substance use disorders across the lifespan, but cannot dismiss such a possibility. Another potential explanation would be early censoring of older adults with late onset BD. If they were to die earlier than those with early onset, this could bias the association with incident use of substances. However, we have previously shown that older adults with longstanding $(\geq 15$ years $)$ and brief history of $\mathrm{BD}(<5$ years $)$ have similar mortality rates (Almeida et al., 2016), which suggests that survivorship bias is an unlikely explanation for our results. Finally, it is possible that people use different adaptive mechanisms to cope with the onset of $\mathrm{BD}$ as they age, so that men with late onset of symptoms would be less likely to use substances for that purpose. Projection, regression and externalisation, for example, are strategies that are more commonly used by younger than older adults to deal with stress (Diehl et al., 1996), and these changes may play a role on whether people choose to use substances in a way that ultimately leads to the development of a disorder. Regardless the mechanism, it is clear that 
older men with onset of BD before age 60 years are at greater risk of prevalent and incident substance use disorders, whereas the risk is much lower among those with late illness onset.

These findings suggest that older people with BD may benefit from a detailed assessment of the use of substances, and health professionals ought to remain alert to the fact that this is a population at risk (Hartz et al., 2014), particularly when illness onset occurs in early adult life. The use of substances has an unfavourable impact on the course of BD and vice-versa (Farren et al., 2013; Strakowski et al., 2005), so that management plans should address both conditions simultaneously. Our findings also suggest that grouping BD into early and late onset is clinically useful and may have prognostic implications that extend beyond the increased risk of dementia (Almeida et al., 2016). 


\section{REFERENCES}

ABS, 2015. Migration, Australia, 2013-14 (3412.0). Australian Bureau of Statistics, Canberra: Australia.

Almeida, O.P., McCaul, K., Hankey, G.J., Yeap, B.B., Golledge, J., Flicker, L., 2016. Risk of dementia and death in community-dwelling older men with bipolar disorder. Br J Psychiatry 209, 121-126.

Breslow, N.E., Lubin, J.H., Marek, P., Langholz, B., 1983. Multiplicative models and cohort analysis. J Am Stat Assoc 78, 1-12.

Cardoso, B.M., Kauer Sant'Anna, M., Dias, V.V., Andreazza, A.C., Cereser, K.M., Kapczinski, F., 2008. The impact of co-morbid alcohol use disorder in bipolar patients. Alcohol 42, 451-457.

Cerami, C., Marcone, A., Galimberti, D., Villa, C., Scarpini, E., Cappa, S.F., 2011. From genotype to phenotype: two cases of genetic frontotemporal lobar degeneration with premorbid bipolar disorder. J Alzheimers Dis 27, 791-797.

Clemens, S.L., Matthews, S.L., Young, A.F., Powers, J.R., 2007. Alcohol consumption of Australian women: results from the Australian Longitudinal Study on Women's Health. Drug Alcohol Rev 26, 525-535.

Coryell, W., Fiedorowicz, J., Leon, A.C., Endicott, J., Keller, M.B., 2013. Age of onset and the prospectively observed course of illness in bipolar disorder. J Affect Disord 146, 34-38.

Dalton, E.J., Cate-Carter, T.D., Mundo, E., Parikh, S.V., Kennedy, J.L., 2003. Suicide risk in bipolar patients: the role of co-morbid substance use disorders. Bipolar Disord 5, 58-61.

Depp, C.A., Jeste, D.V., 2004. Bipolar disorder in older adults: a critical review. Bipolar Disord 6, 343-367.

Diehl, M., Coyle, N., Labouvie-Vief, G., 1996. Age and sex differences in strategies of coping and defense across the life span. Psychol Aging 11, 127-139.

Farren, C.K., Snee, L., Daly, P., McElroy, S., 2013. Prognostic factors of 2-year outcomes of patients with comorbid bipolar disorder or depression with alcohol dependence: importance of early abstinence. Alcohol Alcohol 48, 93-98.

Floris, G., Borghero, G., Cannas, A., Stefano, F.D., Murru, M.R., Corongiu, D., Cuccu, S., Tranquilli, S., Marrosu, M.G., Chio, A., Marrosu, F., 2013. Bipolar affective disorder preceding frontotemporal dementia in a patient with C9ORF72 mutation: is there a genetic link between these two disorders? J Neurol 260, 1155-1157.

Goldstein, B.I., Herrmann, N., Shulman, K.I., 2006. Comorbidity in bipolar disorder among the elderly: results from an epidemiological community sample. Am J Psychiatry 163, 319-321.

Hartz, S.M., Pato, C.N., Medeiros, H., Cavazos-Rehg, P., Sobell, J.L., Knowles, J.A., Bierut, L.J., Pato, M.T., Genomic Psychiatry Cohort, C., 2014. Comorbidity of severe psychotic disorders with measures of substance use. JAMA Psychiatry 71, 248-254. 
Hjorthoj, C., Ostergaard, M.L., Benros, M.E., Toftdahl, N.G., Erlangsen, A., Andersen, J.T., Nordentoft, M., 2015. Association between alcohol and substance use disorders and all-cause and cause-specific mortality in schizophrenia, bipolar disorder, and unipolar depression: a nationwide, prospective, register-based study. Lancet Psychiatry 2, 801-808.

Holman, C.D., Bass, A.J., Rosman, D.L., Smith, M.B., Semmens, J.B., Glasson, E.J., Brook, E.L., Trutwein, B., Rouse, I.L., Watson, C.R., de Klerk, N.H., Stanley, F.J., 2008. A decade of data linkage in Western Australia: strategic design, applications and benefits of the WA data linkage system. Aust Health Rev 32, 766-777.

Hunt, G.E., Malhi, G.S., Cleary, M., Lai, H.M., Sitharthan, T., 2016. Prevalence of comorbid bipolar and substance use disorders in clinical settings, 1990-2015: Systematic review and metaanalysis. J Affect Disord 206, 331-349.

Jablensky, A.V., Morgan, V., Zubrick, S.R., Bower, C., Yellachich, L.A., 2005. Pregnancy, delivery, and neonatal complications in a population cohort of women with schizophrenia and major affective disorders. Am J Psychiatry 162, 79-91.

Merikangas, K.R., Jin, R., He, J.P., Kessler, R.C., Lee, S., Sampson, N.A., Viana, M.C., Andrade, L.H., Hu, C., Karam, E.G., Ladea, M., Medina-Mora, M.E., Ono, Y., Posada-Villa, J., Sagar, R., Wells, J.E., Zarkov, Z., 2011. Prevalence and correlates of bipolar spectrum disorder in the world mental health survey initiative. Arch Gen Psychiatry 68, 241-251.

Meyer, T.D., McDonald, J.L., Douglas, J.L., Scott, J., 2012. Do patients with bipolar disorder drink alcohol for different reasons when depressed, manic or euthymic? J Affect Disord 136, 926-932.

O'Connell, H., Chin, A.V., Cunningham, C., Lawlor, B., 2003. Alcohol use disorders in elderly people--redefining an age old problem in old age. BMJ 327, 664-667.

Regier, D.A., Farmer, M.E., Rae, D.S., Locke, B.Z., Keith, S.J., Judd, L.L., Goodwin, F.K., 1990. Comorbidity of mental disorders with alcohol and other drug abuse. Results from the Epidemiologic Catchment Area (ECA) Study. JAMA 264, 2511-2518.

Sajatovic, M., Blow, F.C., Ignacio, R.V., 2006. Psychiatric comorbidity in older adults with bipolar disorder. Int J Geriatr Psychiatry 21, 582-587.

Sajatovic, M., Strejilevich, S.A., Gildengers, A.G., Dols, A., Al Jurdi, R.K., Forester, B.P., Kessing, L.V., Beyer, J., Manes, F., Rej, S., Rosa, A.R., Schouws, S.N., Tsai, S.Y., Young, R.C., Shulman, K.I., 2015. A report on older-age bipolar disorder from the International Society for Bipolar Disorders Task Force. Bipolar Disord 17, 689-704.

Schouws, S.N., Comijs, H.C., Stek, M.L., Dekker, J., Oostervink, F., Naarding, P., van der Velde, I., Beekman, A.T., 2009. Cognitive impairment in early and late bipolar disorder. Am J Geriatr Psychiatry 17, 508-515.

Strakowski, S.M., DelBello, M.P., Fleck, D.E., Adler, C.M., Anthenelli, R.M., Keck, P.E., Jr., Arnold, L.M., Amicone, J., 2005. Effects of co-occurring alcohol abuse on the course of bipolar disorder following a first hospitalization for mania. Arch Gen Psychiatry 62, 851-858.

Swendsen, J., Conway, K.P., Degenhardt, L., Glantz, M., Jin, R., Merikangas, K.R., Sampson, N., Kessler, R.C., 2010. Mental disorders as risk factors for substance use, abuse and dependence: results from the 10-year follow-up of the National Comorbidity Survey. Addiction 105, 1117-1128. 
Whiteford, H.A., Degenhardt, L., Rehm, J., Baxter, A.J., Ferrari, A.J., Erskine, H.E., Charlson, F.J., Norman, R.E., Flaxman, A.D., Johns, N., Burstein, R., Murray, C.J., Vos, T., 2013. Global burden of disease attributable to mental and substance use disorders: findings from the Global Burden of Disease Study 2010. Lancet 382, 1575-1586. 
Table 1. Association between substance use disorders and bipolar disorder according to age at onset.

\begin{tabular}{|c|c|c|c|}
\hline & Not Bipolar & \multicolumn{2}{|c|}{ Bipolar Disorder: Age at Onset } \\
\hline & $\mathrm{N}=37923$ & $\begin{array}{c}<60 \text { years } \\
\mathrm{N}=175\end{array}$ & $\begin{array}{c}\geq 0 \text { years } \\
\mathrm{N}=75\end{array}$ \\
\hline & mean $\pm \mathrm{SD}$ & mean $\pm \mathrm{SD}$ & mean $\pm \mathrm{SD}$ \\
\hline Age (years) & $72.5 \pm 4.6$ & $71.3 \pm 4.3$ & $74.5 \pm 4.4$ \\
\hline & $\mathrm{n}(\%)$ & $\mathrm{n}(\%)$ & $\mathrm{n}(\%)$ \\
\hline Alcohol use disorder & $1508(4.0)$ & $26(14.9)$ & $7(9.3)$ \\
Crude OR (95\%CI) & 1 (reference) & $4.21(2.77,6.41)$ & $2.49(1.14,5.42)$ \\
Adjusted OR (95\%CI) & 1 (reference) & $3.87(2.52,5.93)$ & $2.38(1.08,5.25)$ \\
\hline Other substance use disorder & $6007(15.8)$ & $49(28.0)$ & $14(18.7)$ \\
Crude OR (95\%CI) & 1 (reference) & $2.07(1.48,2.88)$ & $1.22(0.68,2.18)$ \\
Adjusted OR (95\%CI) & 1 (reference) & $2.07(1.43,3.01)$ & $1.10(0.58,2.09)$ \\
\hline
\end{tabular}

OR: odds ratio compared relative to men without bipolar disorder; $95 \%$ CI: $95 \%$ confidence interval of the odds ratio. Adjusted for age (in years) and for prevalent diabetes, hypertension, ischaemic heart disease and stroke. 


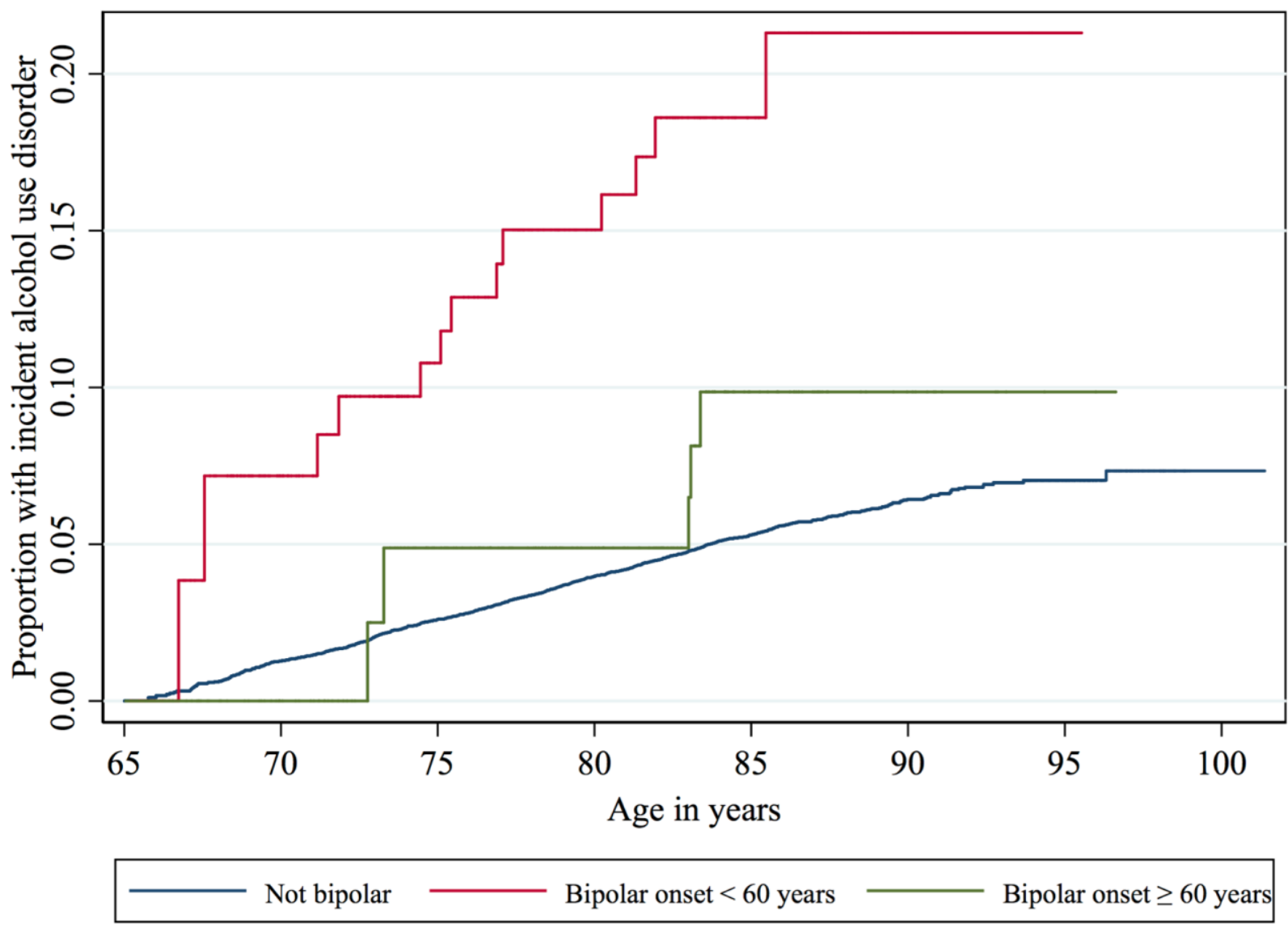

Figure 1. The figure depicts the proportion of older men diagnosed for the first time with an alcohol use disorder according to their age at the time (excludes 1541 prevalent cases; mean \pm SD time of follow up: $11.3 \pm 4.5$ years). 